\begin{tabular}{c|c|c|}
\hline \hline SELECCIONES MATEMÁTICAS \\
Universidad Nacional de Trujillo \\
ISSN: $2411-1783$ (Online) \\
2019; Vol.06(2): $140-147$.
\end{tabular}

\title{
On the numerical solution of a rising sphere in a Newtonian fluid with temperature-dependent viscosity.
}

\section{Sobre la solución numérica de una esfera ascendente en un fluído Newtoniano con viscosidad dependiente de la temperatura.}

\author{
Marcos Zambrano F**
}

Received, Jul. 14, 2019

Accepted, Nov. 11, 2019

How to cite this article:

Zambrano, M. On the numerical solution of a rising sphere in a Newtonian fluid with temperature-dependent viscosity. Selecciones Matemáticas. 2019; 6(2):140-147. http://dx.doi.org/10.17268/sel . mat.2019.02.1

\begin{abstract}
In this work, we present some numerical results about the problem of a rising hot solid sphere immersed in a Newtonian fluid which viscosity depends on the temperature. The model formulated to solve the problem considers two dimensionless parameters: The Peclet number, Pe and a parameter related with the viscosity, $\epsilon$. Small and large variations on $\epsilon$ lead to interesting results segregated into two regimes which exhibit an asymptotic structure. To carry out the computations to solve the proposed model, the element finite method was used along with a non-slip boundary condition for the contact surface between the sphere and the fluid and the results obtained were compared to those shown recently in papers related wherein contact surface has a slip-boundary condition prescribed.
\end{abstract}

Keywords. Newtonian fluid, asymptotic structure, element finite method, contact surface.

Resumen

En este trabajo, presentamos algunos resultados numéricos sobre el problema de una esfera solida caliente ascendente que se encuentra dentro de un fluído newtoniano cuya viscosidad depende de la temperatura. El modelo formulado para resolver el problema considera dos parámetros adimensionales: El número de Peclet, Pe y un parámetro relacionado con la viscosidad, $\epsilon$. Las pequeñas y grandes variaciones sobre $\epsilon$ conducen a interesantes resultados que son divididas en dos regímenes los cuales presentan una estructura asintótica. Para llevar a cabo los cálculos a fin de resolver el modelo propuesto, el método de elemento finito fue usado junto con una condición de frontera no-slip para la superficie de contacto entre la esfera y el fluído y los resultados fueron comparados a aquellos recientemente mostrados en artículos relacionados en donde la superficie de contacto tiene prescrita una condición de frontera slip.

Palabras clave. Fluído newtoniano, estructura asintótica, método del elemento finito, superficie de contacto.

1. Introduction. One important problem found in Geological science is related to the ascent velocity of magmatic diapirs which rise from the depth of the Earth's mantle due to several factors. Geological evidence suggests that these ascending diapirs are bouyant bodies travelling from the Earth's mantle to the Earth's crust how it was explained by Ribe in [1]. Since the rising of the diapir is a complex process, it turns out to be useful to approach this phenomenon through the study of a simplified mathematical model that takes into account some essential features in such a way that to predict some quantities in which we are interested is possible by solving the mentioned model. In this sense, Marsh in [2] proposed a simplified mathematical model, which consists of

*Dpto. Académico de Ciencias Básicas, Universidad Nacional de Barranca - UNAB, Lima-Perú (mzambrano2019@unab.edu • pe). 
a sphere of radius $a$ whose temperature is constant $T_{0}$ and is moving upwards at constant velocity $V$ through a Newtonian fluid whose temperature is $T_{\infty}<T_{0}$ away from the sphere. One key aspect of this model is that the viscosity of the surrounding fluid depends strongly on the temperature [2]. The problem of a rising sphere in a Newtonian fluid was studied by Hadamard [3] and Rybczynski [4] who found analytical solutions for such problem although in isothermal conditions for the fluid. These results formed the background for the same phenomenon in nonisothermal conditions as shown by Acrivos and Goddard in [5] and the analysis of the heat transfer shown by Acrivos and Taylor in [6]. It is important to mention that the rising of a sphere have been a subject for many researchers since this case can occur in the problem of a rising bubble explained by Batchelor in [7] and also in the ascent of magmatic diapir found in [8] which is the subject of the present paper.

Recently, Vynnycky and O'Brien [9] revisited the problem of the rising magmatic diapir and they obtained asymptotic and numerical results by considering in their model a zero-traction surface as shown in [9]. Thus, numerical analysis is a powerful tool to obtain details of the flow of the fluid surrounding the sphere, which would be difficult to retrieve through experiments as mentioned in [10].

The layout of the paper is the following; in section 2, is formulated the mathematical model, in which, viscosity is ruled by a power law, since it is suitable as mentioned in [11]; in section 3, the variables are nondimensionalized and from this, an equation in which the ascending velocity of the spherical diapir is clearly expressed; in section 4 , the data usually used in the simulations, as in [11], are mentioned and some situations of the model based on the values of the Peclet number $P e$ are presented; details of the numerical implementation are specified in section 5; in section 6 , the results obtained in the present paper are shown, in which, an asymptotic structure is revealed and the conclusions are drawn in section 7.

2. Mathematical formulation. Consider a sphere, with density $\rho_{s}$, is rising at speed $V$, through a Newtonian fluid whose density is $\rho_{f}$, with $\rho_{f}>\rho_{s}$ and whose viscosity depends on temperature and is given by the power Law, usually known as Arrhenius Law,

$$
\mu=A \exp (E / \mathcal{R} T)
$$

where $A, E, \mathcal{R}, T$ are respectively a constant, the activation energy, the universal gas constant and the temperature of the fluid.

In cylindrical $(r, \phi, \varphi)$ coordinates, the governing equations of the momentum and continuity equations, in a steady-state are given by

$$
\begin{aligned}
-\frac{\partial p}{\partial r}+\mu\left(\frac{1}{r} \frac{\partial}{\partial r}\left\{r \frac{\partial u_{r}}{\partial r}\right\}+\frac{\partial^{2} u_{r}}{\partial z^{2}}-\frac{u_{r}}{r^{2}}\right) & =0, \\
-\frac{\partial p}{\partial z}+\mu\left(\frac{1}{r} \frac{\partial}{\partial r}\left\{r \frac{\partial u_{z}}{\partial r}\right\}+\frac{\partial^{2} u_{z}}{\partial z^{2}}\right) & =0, \\
\frac{1}{r} \frac{\partial}{\partial r}\left\{r u_{r}\right\}+\frac{\partial u_{z}}{\partial z} & =0,
\end{aligned}
$$

where $u_{r}, u_{z}$ are respectively the horizontal and vertical velocity components, $p$ is the pressure and $\mu$ is the viscosity. Also, the heat transfer equation is given by

$$
\rho_{f} c_{p}\left(u_{r} \frac{\partial T}{\partial r}+u_{z} \frac{\partial T}{\partial z}\right)=k\left(\frac{1}{r} \frac{\partial}{\partial r}\left\{r \frac{\partial T}{\partial r}\right\}+\frac{\partial^{2} T}{\partial z^{2}}\right)
$$

where $\rho_{f}, c_{p}, k$ are respectively, the density, the heat capacity and the thermal conductivity of the fluid. Since the conditions at the sphere differ from those away from sphere, we define

$$
\Gamma_{\beta}=\{(r, z): r=\beta \cos \phi, z=\beta \sin \phi, \forall \phi \in[-\pi, \pi]\} .
$$

With respect to the boundary conditions, on $\Gamma_{a}$,

$$
u_{r}=0, u_{z}=0, T=T_{h}
$$

and as $\beta \rightarrow \infty$,

$$
u_{r}=0, u_{z}=V, T=T_{\infty}
$$

on $\Gamma_{\beta}$, where $T_{\infty}<T_{h}$. In figure 2.1 is depicted a schematic of the rising sphere, whose radius is $a$ and is rising at velocity $V$. Also, the temperature is $T=T_{h}$ on $\Gamma_{a}$. 
Our purpose is to find the ascent velocity $V$ at which the sphere, immersed in the fluid, is rising; and such velocity is determined by a force balance between the buoyancy force of the sphere given by

$$
F_{B}=\frac{4 \pi a^{3}}{3}\left(\rho_{f}-\rho_{s}\right) g
$$

where $g$ is acceleration due to gravity and the drag force given by

$$
F_{D}=\int_{0}^{2 \pi} \int_{-\pi}^{\pi} \mathcal{D} r \mathrm{~d} \phi \mathrm{d} \varphi
$$

where

$$
\mathcal{D}=\mu\left(\frac{\partial u_{z}}{\partial r}+\frac{\partial u_{r}}{\partial z}\right) \cos \phi+\left(-p+2 \mu \frac{\partial u_{z}}{\partial z}\right) \sin \phi .
$$

Such force balance allows to obtain the equation

$$
F_{B}=F_{D}
$$

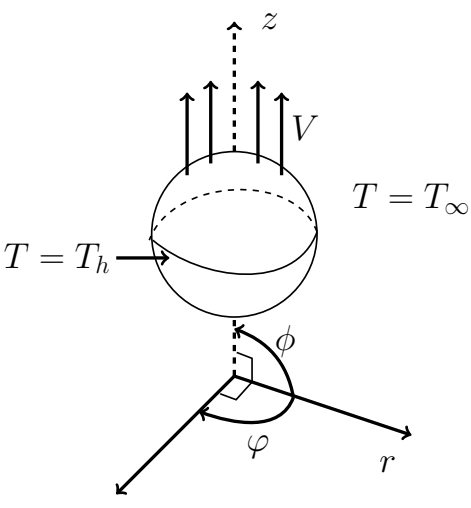

FIGURE 2.1. Schematic of the rising sphere

3. Nondimensionalization. Using the nondimensionalization

$$
R=\frac{r}{a}, Z=\frac{z}{a}, U_{R}=\frac{u_{r}}{V}, U_{Z}=\frac{u_{z}}{V}, P=\frac{p}{[\mu] V / a}, \theta=\frac{T}{T_{h}}, \bar{\mu}=\frac{\mu}{[\mu]},
$$

where $[\mu]$ is the viscosity scale given by

$$
[\mu]=A \exp \left(\frac{1}{\epsilon}\right)
$$

equations (2.2),(2.3) and (2.4) becomes

$$
\begin{aligned}
-\frac{\partial P}{\partial R}+\bar{\mu}\left(\frac{1}{R} \frac{\partial}{\partial R}\left\{R \frac{\partial U_{R}}{\partial R}\right\}+\frac{\partial^{2} U_{R}}{\partial Z^{2}}-\frac{U_{R}}{R^{2}}\right) & =0 \\
-\frac{\partial P}{\partial Z}+\bar{\mu}\left(\frac{1}{R} \frac{\partial}{\partial R}\left\{R \frac{\partial U_{Z}}{\partial R}\right\}+\frac{\partial^{2} U_{Z}}{\partial Z^{2}}\right) & =0 \\
\frac{1}{R} \frac{\partial}{\partial R}\left\{R U_{R}\right\}+\frac{\partial U_{Z}}{\partial Z} & =0 .
\end{aligned}
$$

Also (2.5) turns into

$$
P e\left(U_{R} \frac{\partial \theta}{\partial R}+U_{Z} \frac{\partial \theta}{\partial Z}\right)=\frac{1}{R} \frac{\partial}{\partial R}\left\{R \frac{\partial \theta}{\partial R}\right\}+\frac{\partial^{2} \theta}{\partial Z^{2}},
$$

where $P e$ denotes the Peclet number given by

$$
P e=\frac{V a}{k / \rho_{f} c_{p}}
$$


and the related-term to the viscosity becomes

$$
\bar{\mu}=\exp \left(\frac{1-\theta}{\epsilon \theta}\right),
$$

where $\epsilon=\mathcal{R} T_{h} / E$ is called usually called the viscosity variation as mentioned in [9].

For the boundary conditions, we have from (2.7)

$$
U_{R}=0, U_{Z}=0, \theta=1
$$

on $\Gamma_{1}$, and from (2.8)

$$
U_{R}=0, U_{Z}=1, \theta=\theta_{\infty}
$$

on $\Gamma_{\beta}$ as $\beta \rightarrow \infty$, where $\theta_{\infty}=T_{\infty} / T_{h}$.

Figure 3.1 shows a schematic of the boundary conditions of the equations (3.3), (3.4), (3.5) and (3.6). Also, a symmetry boundary condition is prescribed due to the geometry of the domain which contains the fluid.

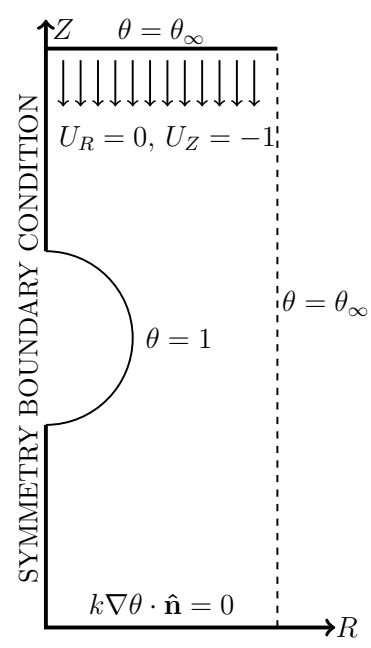

FIGURE 3.1. Schematic of the Boundary conditions for the Sphere.

On the other hand, equation (2.12) becomes, explicitly,

$$
-\frac{4 \pi a^{3}}{3}\left(\rho_{f}-\rho_{s}\right) g+[\mu] V \int_{0}^{2 \pi} \int_{-\pi}^{\pi} \tilde{\mathcal{D}} \mathrm{d} \phi \mathrm{d} \varphi=0,
$$

where

$$
\tilde{\mathcal{D}}=\bar{\mu}\left(\frac{\partial U_{Z}}{\partial R}+\frac{\partial U_{R}}{\partial Z}\right) \cos \phi+\left(-P+2 \bar{\mu} \frac{\partial U_{Z}}{\partial Z}\right) \sin \phi .
$$

Now, once obtained (3.11), an expression in which, the velocity-term $V$ is clearly mentioned, we are allowed to elaborate a strategy to calculate it.

4. Analysis. Using data, presented in [11],

$$
\begin{gathered}
\rho_{f} \sim 2850 \mathrm{kgm}^{-3}, \rho_{s} \sim 2300 \mathrm{kgm}^{-3}, c_{p} \sim 1000 \mathrm{Jkg}^{-1} \mathrm{~K}^{-1}, k \sim 2 \mathrm{Wm}^{-1} \mathrm{~K}^{-1}, \\
a \sim 3000 \mathrm{~m}, T_{h} \sim 1100 \mathrm{~K}, E \sim 142000 \mathrm{Jmol}^{-1}, \mathcal{R}=8.3145 \mathrm{Jmol}^{-1} \mathrm{~K}^{-1}, A=2.3 \times 10^{11} \mathrm{~Pa} \mathrm{~s} .
\end{gathered}
$$

It was obtained

$$
\epsilon \sim 0.0644,[\mu] \sim 1.2 \times 10^{23} .
$$

Although, initially $\epsilon$ is small, this value can change due to several factor, for instance, the temperature of the sphere, etc. Thus, the analysis will be carried out by considering two segregated regions

(A) $\epsilon \ll 1$. Significant conduction in the heat transfer and the advection is negligible.

(B) $\epsilon \gg 1$. The advection is predominant over the conduction. 
4.1. $\epsilon \ll 1$. From (3.6), we obtain

$$
\frac{1}{R} \frac{\partial}{\partial R}\left\{R \frac{\partial \theta}{\partial R}\right\}+\frac{\partial^{2} \theta}{\partial Z^{2}}=0,
$$

subject to the boundary conditions, $\theta=1$ on $\Gamma_{1}$ and $\theta \rightarrow \theta_{\infty}$ on $\Gamma_{\beta}$ as $\beta \rightarrow \infty$.

4.2. $\epsilon \gg 1$. Also, from (3.6), we have

$$
U_{R} \frac{\partial \theta}{\partial R}+U_{Z} \frac{\partial \theta}{\partial Z}=0
$$

subject to the boundary condition $\theta=1$ on $\Gamma_{1}$.

5. Numerical implementation. Equations (3.3)-(3.6) are solved using the finite-element technique with $P_{2}-$ $P_{1}$ elements for the velocity and pressure respectively. It was used COMSOL Multiphysics software (https://www.comsol.com), to generate the mesh in the domain and to solve the equations of the model subsequently. Figure 5.1 shows the mesh of the region to be solved. The domain was set for $R_{\infty}=12$, hence $0 \leq R \leq R_{\infty}$ and setting $Z_{\infty}=12$, so that $-Z_{\infty} \leq Z \leq Z_{\infty}$. Also on the boundary of the domain away from sphere is prescribed $\theta=\theta_{\infty}$ and on $\Gamma_{1}$ a finer mesh was used with the intention to compute a accurate solution near this boundary.

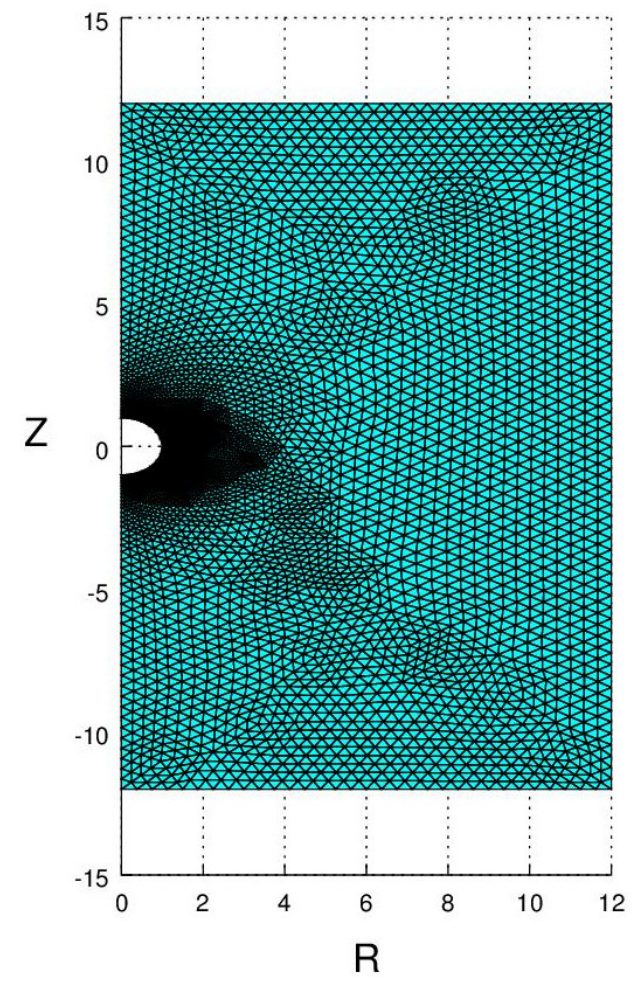

FIGURE 5.1. The mesh of the region to solve equations (3.3-3.6).

To compute the solution and to obtain the velocity $V$ depending on $\epsilon$, initially, it is necessary to prescribe a starting value of $\theta$, say, $\theta_{0}$ to compute the viscosity in the whole domain, so, to compute the solution of (3.3)-(3.5) is possible.

Once computed the distribution of velocities in the domain, it was possible to calculate the solution of equation (3.6), This iterative procedure allows obtain a sequence of the temperature in the domain, whose limit is the distribution of temperature in the domain that along with $U_{R}, U_{Z}$ and $P$ satisfies equations (3.3)-(3.6).

In algorithm 1, the steps to obtain the ascending velocity $V$ given a value of $\epsilon>0$ must be executed sequentially, also, to provide a small value for the tolerance is mandatory due to the accuracy needed to compute the value of $V$.

6. Results. Once applied the algorithm 1 , several results were obtained. In figure 6.1 , the simulations considered $\epsilon \ll 1$ and it is clearly shown an asymptotic behaviour of the Peclet number which tends to 0 .

In figure 6.2, it can seen an increment of the Peclet number when $\epsilon \sim 1$, this region can be considered as a transition region. 

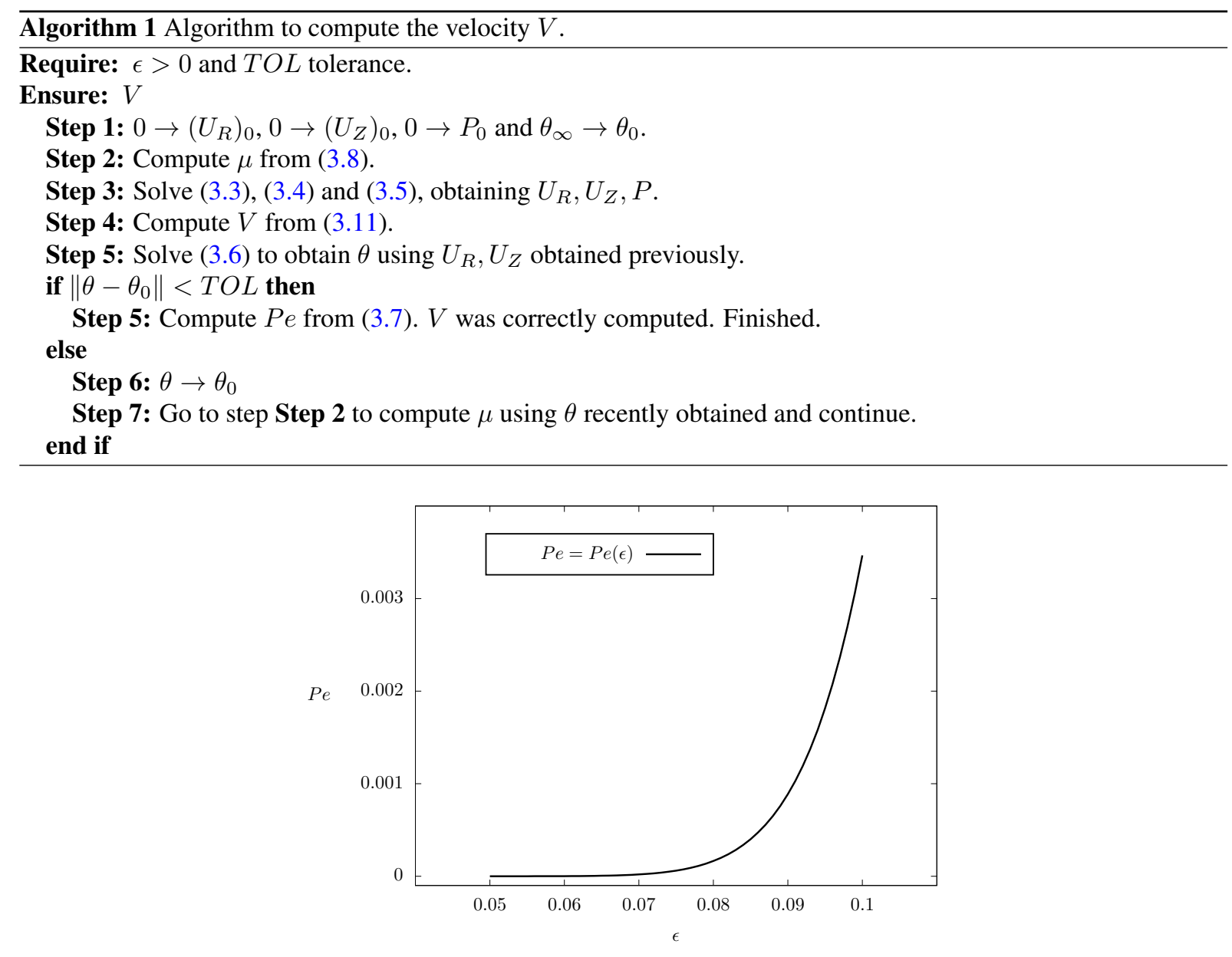

FIGURE 6.1. Peclet number $v s \epsilon$ for small values of $\epsilon$.

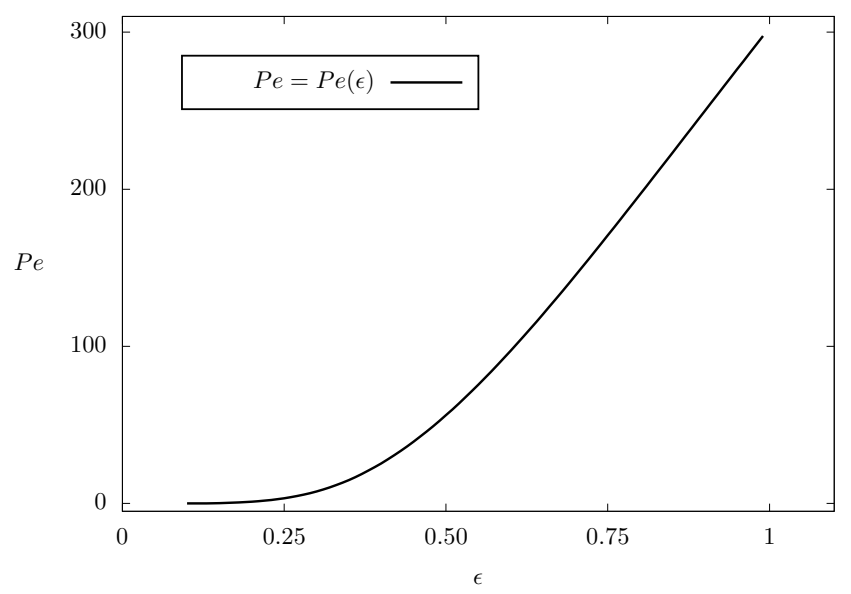

FIGURE 6.2. Peclet number $v$ s $\epsilon$ for values of $\epsilon \sim 1$.

In figure 6.3, the values of Peclet number are large and they were obtained as a result of simulations, in which $\epsilon \gg 1$. Here, it can be observed, clearly, that there exists an asymptotic behaviour for the Peclet number.

In figure 6.4 are shown the values obtained from equation (3.11) once the algorithm 1 has been carried out and the numerical solution of equations (3.3)-(3.6) was obtained for a range of values of $\epsilon$.

Finally, in figure 6.5 are shown isothermal lines for different values of $\epsilon$, in (a), the isothermal lines are parallel lines to the semi-circle shown, in this case, as seen in section 4 for $\epsilon \ll 1$, the mechanism of heat transfer is governed mainly by conduction. When increased the value of $\epsilon$, say, $\epsilon \sim 1$, isothermals change their shape in this region of transition. 


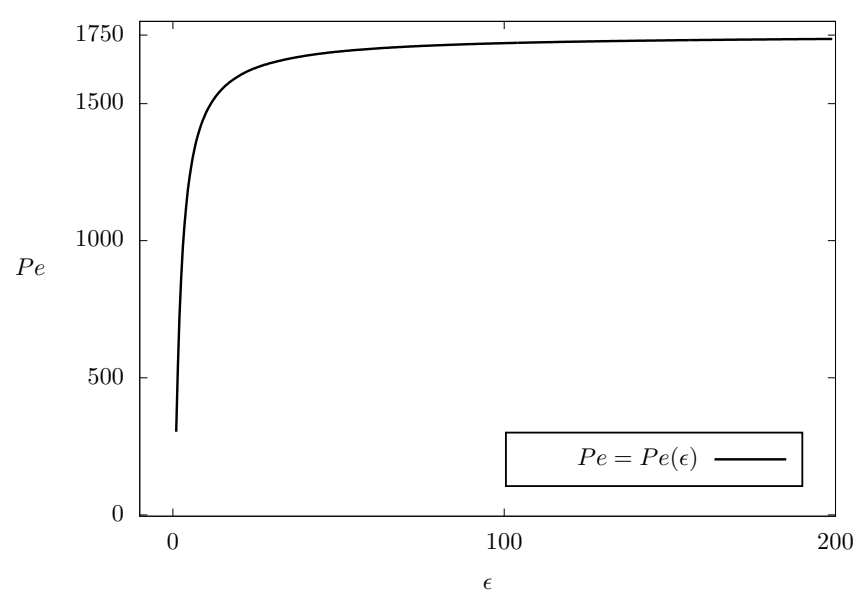

FIGURE 6.3. Peclet number $v$ s $\epsilon$ for large values of $\epsilon$.

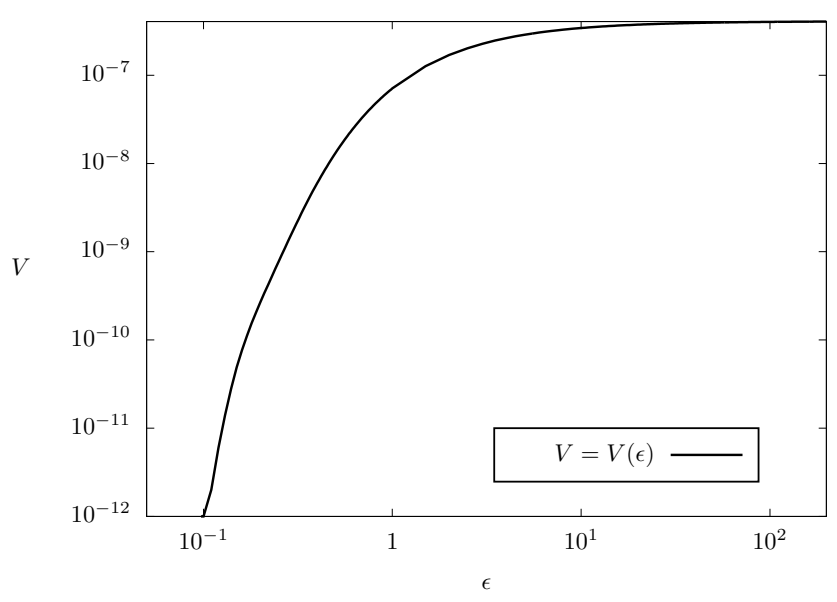

FIGURE 6.4. $V$ velocity $v s \in$ obtained using equation (3.11).

On the other hand, for large values of $\epsilon, \epsilon \gg 1$, isothermal lines are clustered next to the symmetry axis, here, the mechanism of heat transfer is mainly carried out by advection as shown in section 4 .

\section{Conclusions.}

1. The nondimensionalization of the variables, in section 3, allows to obtain equation (3.11), in which, the ascending velocity $V$ was explicitly expressed, allowing to calculate it. In addition, This equation shows a balance between the drag of the sphere and on buoyancy force on it.

2. The values for the viscosity variation $\epsilon$ were segregated into two regions, for $\epsilon \ll 1$ and $\epsilon \gg 1$ and in each region the Peclet number $P e$ (as function of $\epsilon$ ) was computed, from this, in both regions an asymptotic structure was shown. These region was linked by a region, for which, $\epsilon \sim 1$.

3. These results were obtained using the finite-element technique, starting to mesh the region for the computation and solving equations (3.3)-(3.6) via algorithm 1. Also, the mesh was refined near the sphere to obtain accurate results.

4. The isothermal lines for different values of the viscosity variation, $\epsilon$, show the influence of the mechanism of the heat transfer, either by conduction, when $\epsilon \ll 1$, or by convection, when $\epsilon \gg 1$. In the transition region, when $\epsilon \sim 1$, both mechanisms as conduction as convection interact which is expressed in equation (3.6).

8. Acknowledgements. The author thanks Dr. Michael Vynnycky for his patience, unvaluable advices and constant support during several months, in which, the mathematical modelling and numerical simulation on this subject took place.

ORCID and License

Marco Zambrano https://orcid.org/0000-0002-4327-9000.

This work is licensed under the Creative Commons Attribution-NoComercial-ShareAlike 4.0. 

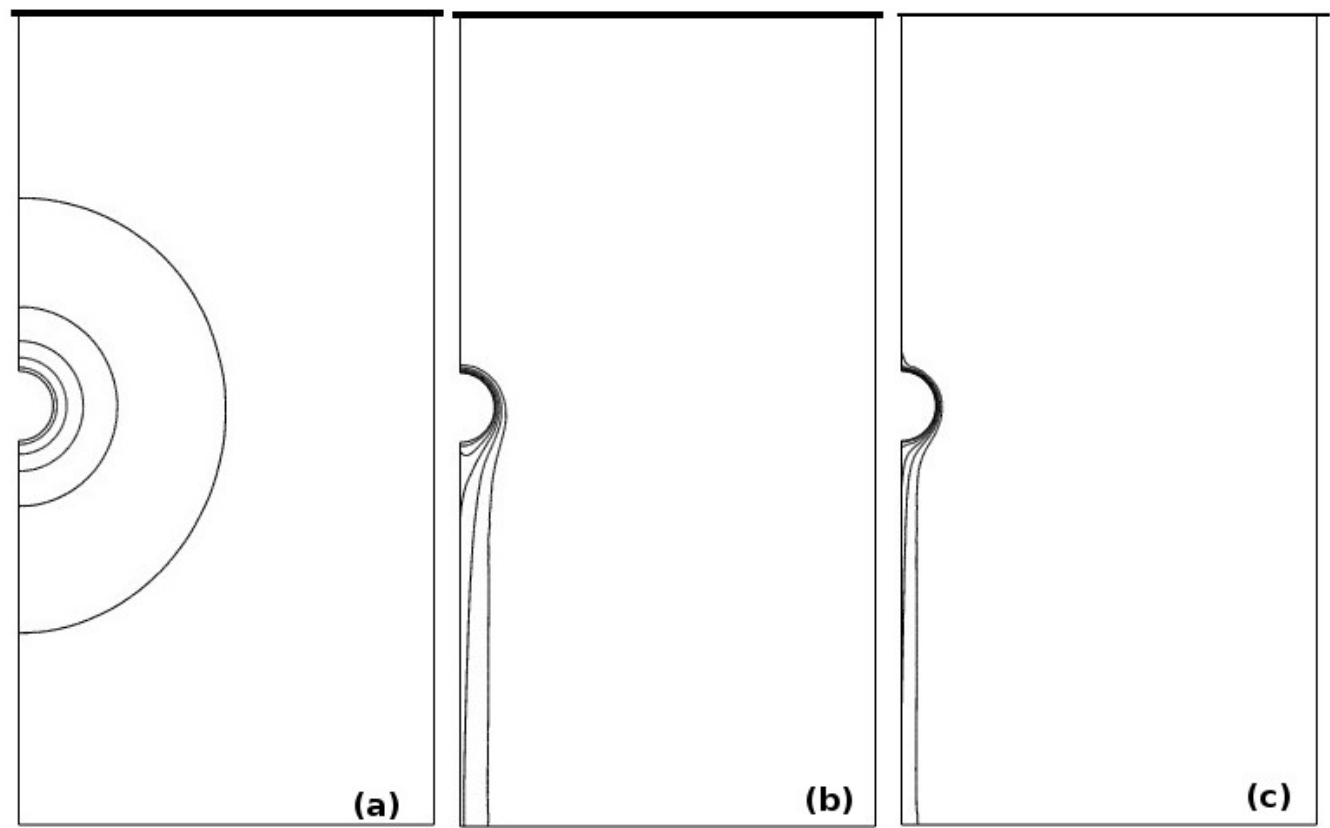

FIGURE 6.5. Isothermal lines for $\theta$ for different values of $\epsilon=0.07(a), \epsilon=0.7(b)$ and $\epsilon=50(c)$.

\section{References}

[1] Ribe, N. M. Diapirism in the Earth's Mantle: Experiments on the motion of a hot sphere in a fluid with temperature-dependent visocity, Journal of Volcanology and Geothermal Research. 1983; (16):221-245.

[2] Marsh, B. D. On the cooling of ascending andesitic magma, Philos. Trans. R. Soc. London; 1978; 288:611-625.

[3] Hadamard, J. Mouvement permanent lent d'une sphere liquide et visqueuse dans un liquid visqueus, C.R. Acad. Sci. 1911; 152:17351738.

[4] Rybczynski, W. Über die fortschreitende ewegung einer flüssigen Kugel in einen zähen Medium, Bull Acad. Sci. 1911; 1:40-46.

[5] Acrivos, A. and Goddard J. D., Asymptotic expansions for laminar forced convection heat and mass transfer. Part 1. Low speed flows, J. Fluid Mech. 1965; 23:273-291.

[6] Acrivos, A. and Taylor T. D., Heat and Mass transfer from single spheres in Stokes flow, Phys, Fluids. 1962; 5:387-394.

[7] Batchelor, G. K., An Introduction to Fluid Dynamics, Cambridge University Press, 1967.

[8] Ansari, M. and Morris S., The effects of a strongly temperature-dependent viscosity on Stokes' draw law: experiments and theory, Journal of Fluid Mechanics. 1985; 159:459-476.

[9] Vynnycky, M. and O'Brien, M. A., The slow, steady ascent of a hot solid sphere in a Newtonian fluid with strongly temperature-dependent viscosity, App. Math. Comp. (2014), 231-253.

[10] Zhang, L. et al, Numerical Simulation of a bubble rising in shear-thinning fluids. J. Non-Newtonian Fluid Mech. 2010; 165:555-567.

[11] Weinberg, R. and Podladchikov Y., Diapiric ascent of magmas through power law crust and mantle, J. Geophys. Res. 1994; B5:95439559. 